\title{
Effects of a vestibular rehabilitation program on workers in the working environment: a pilot study
}

\author{
Isadora Altero Longo ${ }^{(1)}$ \\ Ariane Diane Morais Nunes ${ }^{(1)}$ \\ Clayton Henrique Rocha ${ }^{(1)}$ \\ Fabiana Mara Branco(2) \\ Renata Rodrigues Moreira(2) \\ Ivone Ferreira Neves-Lobo ${ }^{(1)}$ \\ Raquel Aparecida Casarotto(1) \\ Alessandra Giannella Samelli(1)
}

(1) Faculdade de Medicina da Universidade de São Paulo - São Paulo, SP, Brasil.

(2) Hospital Universitário da Universidade de São Paulo - São Paulo, SP, Brasil.

Conflict of interests: Nonexistent

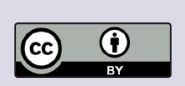

Received on: December 20, 2017 Accepted on: March 29, 2018

Mailing address:

Dra Ivone Ferreira Neves-Lobo Departamento de Fisioterapia,

Fonoaudiologia e Terapia Ocupacional da Faculdade de Medicina da Universidade de São Paulo (FMUSP)

Rua Cipotânea, 51. Cidade Universitária CEP: 05360-160 - São Paulo, São Paulo, Brasil

E-mail: ifneves@usp.br

\section{ABSTRACT}

Objective: to verify the effect of a vestibular rehabilitation program on workers with dizziness from the Division of Nutrition and Dietetics at a University Hospital.

Methods: a total of 13 employees between 42 and 65 years of age participated, of whom, 1 was male, and 12, females. They were evaluated before and after the vestibular rehabilitation program using the Dizziness Handicap Inventory, Work Ability Index and Visual Analog Scale. The program was conducted in groups at the workplace. Statistical analysis was performed using the analysis of variance test for paired factors and the chi-square test.

Results: in the pre- and post-vestibular rehabilitation comparison, significant differences were found in the Dizziness Handicap Inventory and Visual Analogue Scale scores. In the qualitative analysis of the Work Ability Index, an increase was observed in the number of workers who considered their ability to work to have improved.

Conclusion: vestibular rehabilitation conducted in groups and in the working environment was effective in improving dizziness complaint in workers at the Division of Nutrition and Dietetics. It is believed that the positive experience of this program may be extended to other areas.

Keywords: Work; Workers; Dizziness; Rehabilitation; Quality of Life 


\section{INTRODUCTION}

The benefits of actions aimed at improving workers' health have been widely demonstrated, due to their positive implications for the worker and, consequently, for the company. The introduction of activities that help to promote health and improve quality of life in the workplace is a universal practice; however, such actions are normally more focused on musculoskeletal symptoms.

Dizziness is considered one of the most common complaints in clinical practice and is present in between $5 \%$ and $10 \%$ of the world's population. Similarly, it is estimated that the prevalence of dizziness in workers is between 12 and $13 \%{ }^{1}$. Dizziness can be defined as the illusion of motion of the individual himself or the environment that surrounds him $^{2}$ and can be characterized as rotating and non-rotating. The former is usually vestibular in origin and is called vertigo, whereas the second may or may not be vestibular ${ }^{3}$.

Individuals with dizziness may have associated complaints, such as difficulty concentrating, memory loss, fatigue, physical and psychological insecurity, irritability, anxiety, loss of self-confidence, depression and panic, sweating, nausea and vomiting ${ }^{2}$. Such problems may lead to restrictions in social and daily activities, may interfere with work performance and, consequently, affect quality of life ${ }^{4}$. A study of workers with dizziness found that $27 \%$ reported changing jobs and that $21 \%$ stopped working. More than $50 \%$ of the group of workers who continued performing the same work activities were aware that their work efficiency had decreased considerably. Furthermore, it was estimated that in 6 months, the average number of days off due to dizziness was 7.15 days $^{5}$.

Although vertigo is a fairly common complaint, changes are often not detected in vestibular tests due to the labyrinth's hypersensitivity to changes in other organs or bodily systems, generating secondary vestibular changes and little impairment of the vestibular apparatus, even in the presence of vertigo symptoms. The application of validated questionnaires and the obtaining of clinical and family history data by means of anamnesis are essential in terms of diagnosis and defining treatment ${ }^{6}$.

Vestibular rehabilitation (VR) has been an effective method for eliminating or reducing dizziness or vertigo in different populations, with or without vestibular changes identified in vestibular tests 7,8 .

VR consists of a set of exercises aimed at stimulation of the vestibulocochlear, vestibulocervical and vestibulospinal reflexes to accelerate the functional balance recovery process ${ }^{9}$. The central element of this treatment is an exercise program in which eye, head and body movements are stimulated to then stimulate the vestibular system ${ }^{10}$.

Recent studies with VR have shown significant improvements in dizziness in elderly and other populations with chronic vestibular disorders ${ }^{11,12}$. No studies on VR's effectiveness specifically for workers were found in the studied literature, nor were any that have been performed as a workers' group health promotion action or in the working environment.

The Work Ability Index (WAl), already tested and validated for Portuguese, is a tool that has often been used to evaluate how well a worker is and how able he is to perform his duties in respect of the demands of his health status and physical and mental abilities 13. The presence of symptoms and diseases and selfperceived health status is considered the basis for the ability to work ${ }^{14,15}$.

Knowing the effects of dizziness on a workers' quality of life, and considering the effectiveness of VR programs and its being a low-cost method without the need for specialized equipment, it is believed that action focused on vestibular system stimulation can contribute to the improvement of dizziness and, consequently, to quality of life and the ability to work. It is hoped that analysis of the effects of this program will contribute to the development of future group actions in the working environment.

This report describes experiences aimed at analyzing the effect of a VR program in the working environment on workers with dizziness and determining its effect on their perception of ability to work.

\section{METHODS}

The study was approved by the Ethics and Research Committee of Hospital Universitário da Universidade de São Paulo - HUUSP under protocol No. 346,064, respecting Resolution 466/2012 of the National Health Council (Conselho Nacional de Saúde).

\section{Sample}

This was a quasi-experimental study (with no control group), with the participation of 13 individuals aged 42 to 65 with dizziness, 1 male and 12 females, all performing the kitchen assistant role in the Division of Nutrition and Dietetics (DND) of a university hospital. 
After the research was explained to them, all study participants signed informed consent forms.

The DND is the division responsible for the preparation of meals for hospitalized patients and their companions and for the employees, interns and residents at the hospital. The DND has 120 employees, who perform the functions of nutritionist, nutrition technician, cook and kitchen assistant. There is a daily workload of 8 hours, divided into 3 shifts (morning, afternoon and evening), with 12-hour shifts on weekends and holidays.

The DND has existed for over 40 years, and many employees have been there since its inception. Because the activities are heavy and due to aging and chronic diseases, some of these employees now have restrictions regarding the performance of some of their tasks. These restrictions were the reason the DND board requested the evaluation of the section and the development of actions that could improve working conditions.

During this evaluation, 23 workers who exercised the functions of cook or kitchen assistant had restrictions regarding performing their work. For the vast majority, the restriction was related to musculoskeletal complaints, but dizziness and the occurrences of falls, at work and at home, were also reported. Therefore, the need was observed to work to improve balance among this group to decrease dizzy spells and episodes of falls.

The selection of employees to participate in the study was conducted in stages. First, a preliminary screening was performed using anamneses of these 23 DND workers to verify the presence of dizziness within the past 12 months and/or outside of the working environment. Ten workers who did not have this symptom, or who could not participate in the program because they were on vacation, were excluded. It should be noted that the workers underwent periodic medical supervision.

The second stage included the 13 workers who had experienced dizziness during the previous 12 months but who had not received treatment for vertigo and who had no obvious neurological or psychiatric diseases. In addition, the Dix-Hallpike maneuver was performed to rule out Benign Paroxysmal Positional Vertigo (BPPV) ${ }^{10}$.

\section{Procedures}

The workers involved were given instructions about each procedure and were told that all of the activities would be performed in the comfort area of the section, at a pre-established time agreed between the supervisor and worker during the working day. As the study proposal was to perform VR in the workplace, procedures that were simple and easy to use and reproduce were chosen. Employees who reported frequent dizziness were instructed to seek the hospital's otorhinolaryngology service for evaluation and medical management.

All participants were evaluated with the following tools, before and after implementation of the VR program:

1. Dizziness Handicap Inventory (DHI), translated and validated for the Brazilian Portuguese language. This inventory evaluates the perception of the effects caused by dizziness and addresses the physical, emotional and functional aspects. Its scores range from 0 to 100 and are classified as follows: from $0-25-$ no handicap; 26-50 - slight handicap; 51-75 - moderate handicap and 76-100 - severe handicap ${ }^{16}$. The greater the handicap, the greater the harm caused by dizziness.

2. Work Ability Index (WAI), translated and validated for the Brazilian Portuguese language, which evaluates the physical, mental and social skills associated with work, according to the individual's perception ${ }^{13,17}$. The scores range from 0 to 49 , with 7 to 27 classified as low ability to work, $28-36$ as moderate ability, $37-43$ as good ability and 44-49 as excellent ability. A reduction of 18 points in the sum of the scales is considered an improvement criterion.

3. Visual Analogue Scale (VAS) for the dizziness symptom, in which the symptom was graded by the worker from 0 to 10 , where 0 corresponds to the absence of dizziness and 10 is the greatest dizziness ever felt.

The VR program was applied by resident audiologists and physical therapists of the hospital and based on the protocols of Cawthorne (1944) ${ }^{18}$ and Cooksey (1946) ${ }^{19}$. The exercise protocols aim to associate eye, head and body movements in different positions, at different speeds and alternating the presence of visual compensation ${ }^{18-20}$. The program consisted of 8 sessions, each lasting an average of 20 minutes. The number of sessions was defined according to the average of the various studies reviewed ${ }^{4,10}$; each meeting was held at a time agreed by the division coordination because the meetings were held during working hours within the workplace.

Groups were formed of 2 or 3 workers with similar degrees of dizziness, on the division's morning and afternoon shifts. All workers underwent the same 
exercise program, and in each session, the degree of difficulty in performing the exercises was rated, progressing to a new exercise when the previous one was completed adequately. Participants were advised about the importance of attending every session and performing the exercises at home, as this adherence would facilitate effectiveness of the VR program.

A folder was given to the workers explaining the exercises and providing guidelines for the daily performance of the exercises at home. To monitor the implementation of home exercises, the workers received a notebook, where they were to note whether they performed the exercises and the degree of dizziness that they felt. This degree was rated from 0 to 4 , where 0 equaled "no dizziness" and 4 equaled "unbearable dizziness". The worker was told to stop the exercise at home if the level was greater than 2. This classification was used to direct the exercises performed every week according to the perception of dizziness reported by the group, with no other purpose than to serve as a parameter of the exercises performed at home.

After carrying out 8 meetings, in addition to the questionnaires mentioned above, a dizziness selfassessment was performed based on the following question: "Do you think that participating in the program has improved your dizziness?" Responses were given according to the following alternatives: "has not improved," "improved," "improved slightly", "improved a lot". Finally, an open space was left for criticisms and/ or suggestions.

Descriptive analyses were performed, obtaining the mean values and standard deviations and percentages for the pre- and post-VR questionnaires. The analysis of variance for one paired factor and the Chi-square test (Fisher's exact test) were performed, considering values with $p \leq 0.05$ to be statistically significant. In addition, qualitative analyses were performed by means of open questions about improvements to the participants' dizziness after the VR program.

\section{RESULTS}

Table 1 shows the characterization of the sample and dizziness reported by the study participants.

The results of the DHI show a decrease, for most participants, on the 3 handicap subscales after completion of the VR, with statistically significant differences in the pre- and post-VR score comparison on the 3 subscales (Table 2 ).

Table 3 shows a statistically significant difference between pre- and post-VR scores for VAS. No statistically significant difference was found for the WAI questionnaire.

In the WAI and DHI qualitative analysis (Table 4), the categories "low" and "moderate" and "good" and "excellent" were grouped in the WAI questionnaire; and handicaps "none" and "mild" and "moderate" and "severe" were grouped in the $\mathrm{DHI}$ because the number of occurrences in some categories was very small, which would make statistical analysis unfeasible. For this analysis, no statistically significant differences were found regarding the initial and final WAI and DHI classifications.

With respect to performing exercises at home during the program, $66.7 \%$ performed them 1 to 3 days per week, and $33.3 \% 4$ to 7 days a week. When comparing these data with the initial and final DHI scores, no statistically significant difference was observed $(p=0.81)$.

With regard to the perception of dizziness after implementation of the program, when asked, "Do you think that participating in the program has improved your dizziness?", $50 \%$ of participants said that it had "improved a lot", $25 \%$ said it had "improved" and 25\% said it had "improved slightly". No participant gave a negative response. 
Table 1. Sample profile prevalence and characterization of related dizziness complaints

\begin{tabular}{|c|c|c|c|c|c|c|c|c|c|}
\hline Participant & Gender & Age & Function & Rotating & $\begin{array}{c}\text { Non- } \\
\text { rotating }\end{array}$ & Blurred vision & $\begin{array}{l}\text { Instability/ } \\
\text { Imbalance }\end{array}$ & $\begin{array}{c}\text { Fainting } \\
\text { sensation }\end{array}$ & $\begin{array}{l}\text { Scotomas/ } \\
\text { Phosphenes }\end{array}$ \\
\hline P01 & $\mathrm{F}$ & 42 & $\begin{array}{l}\text { Kitchen } \\
\text { assistant }\end{array}$ & Yes & & Yes & Yes & & Yes \\
\hline P02 & M & 56 & Cook & & Yes & Yes & & & \\
\hline P03 & $\mathrm{F}$ & 48 & $\begin{array}{l}\text { Kitchen } \\
\text { assistant }\end{array}$ & Yes & & & Yes & & Yes \\
\hline P04 & $\mathrm{F}$ & 46 & $\begin{array}{l}\text { Kitchen } \\
\text { assistant }\end{array}$ & Yes & & & Yes & Yes & \\
\hline P05 & $\mathrm{F}$ & 51 & $\begin{array}{l}\text { Kitchen } \\
\text { assistant }\end{array}$ & Yes & & & Yes & & Yes \\
\hline P06 & $\mathrm{F}$ & 49 & $\begin{array}{l}\text { Kitchen } \\
\text { assistant }\end{array}$ & Yes & & Yes & Yes & & \\
\hline P07 & $\mathrm{F}$ & 65 & $\begin{array}{l}\text { Kitchen } \\
\text { assistant }\end{array}$ & Yes & & & Yes & & \\
\hline P08 & $\mathrm{F}$ & 51 & $\begin{array}{l}\text { Kitchen } \\
\text { assistant }\end{array}$ & Yes & & & Yes & & \\
\hline P09 & $\mathrm{F}$ & 52 & $\begin{array}{l}\text { Kitchen } \\
\text { assistant }\end{array}$ & Yes & & & Yes & Yes & \\
\hline P10 & $\mathrm{F}$ & 51 & $\begin{array}{l}\text { Kitchen } \\
\text { assistant }\end{array}$ & Yes & & & Yes & & \\
\hline P11 & $\mathrm{F}$ & 51 & $\begin{array}{l}\text { Kitchen } \\
\text { assistant }\end{array}$ & Yes & & & Yes & & \\
\hline P12 & $\mathrm{F}$ & 43 & $\begin{array}{l}\text { Kitchen } \\
\text { assistant }\end{array}$ & & Yes & & Yes & & \\
\hline P13 & $\mathrm{F}$ & 46 & $\begin{array}{c}\text { Kitchen } \\
\text { assistant }\end{array}$ & Yes & & & Yes & & \\
\hline \multicolumn{2}{|c|}{ Mean age } & 50.08 & - & - & - & - & - & - & - \\
\hline \multicolumn{2}{|c|}{ Prevalence (\%) } & - & - & $84.6 \%$ & $15.4 \%$ & $23.1 \%$ & $92.3 \%$ & $15.4 \%$ & $23.1 \%$ \\
\hline
\end{tabular}

Legend: $P_{n}$ : Participant; F: Female; M: Male.

Table 2. Results obtained from analysis of variance for one paired factor, comparing the values obtained from the Dizziness Handicap Inventory (DHI) (physical, functional and emotional subscales and total scale), pre- and post-vestibular rehabilitation program

\begin{tabular}{ccccc}
\hline $\begin{array}{c}\text { DHI } \\
\text { Subscales }\end{array}$ & Evaluation & Mean & SD & P-value \\
\hline Physical & Pre & 12.33 & 4.96 & $0.041^{*}$ \\
& Post & 9 & 4.78 & \multirow{2}{*}{$0.001^{*}$} \\
Functional & Pre & 15.5 & 8.66 & \\
& Post & 10 & 7.43 & $0.046^{*}$ \\
Emotional & Pre & 9.83 & 10.3 & \\
& Post & 5.33 & 8.28 & $0.003^{*}$ \\
Total Scale & Pre & 37.66 & 21.12 & 18.44 \\
\hline
\end{tabular}

Legend: DHI: Dizziness Handicap Inventory; SD: standard deviation; Pre: pre-vestibular rehabilitation program; Post: post-vestibular rehabilitation program. Level of significance adopted: $5 \%$. * statistically significant difference. 
Table 3. Results obtained from analysis of variance for one paired factor, comparing the values obtained from the Visual Analog Scale (VAS) and the Work Ability Index (WAI), pre- and post-vestibular rehabilitation program

\begin{tabular}{ccccc}
\hline & Evaluation & Mean & SD & P-value \\
\hline \multirow{2}{*}{ VAS } & Pre & 5.89 & 1.55 & \multirow{2}{*}{$0.0001^{*}$} \\
& Post & 2.8 & 2.13 & \multirow{2}{*}{0.201} \\
\hline
\end{tabular}

Legend: VAS: Visual Analog Scale; WAI: Work Ability Index; SD: standard deviation; Pre: pre-vestibular rehabilitation program; Post: post-vestibular rehabilitation program. Level of significance adopted: $5 \%$. statistically significant difference.

Table 4. Result of Chi-square test (Fisher's Exact Test) comparing the initial and final scores of the Work Ability Index and the Dizziness Handicap Inventory

\begin{tabular}{ccccc}
\hline & Index & Initial & Final & P Value \\
\hline \multirow{2}{*}{ WAI } & Low/moderate & $91.66 \%$ & $75 \%$ & 0.590 \\
& Good/Excellent & $8.43 \%$ & $25 \%$ & \\
DHI & None/Mild & $66.66 \%$ & $83.33 \%$ & 0.640 \\
& Moderate/Severe & $33.34 \%$ & $16.67 \%$ & \\
\hline
\end{tabular}

Legend: WAl: Work Ability Index; DHI: Dizziness Handicap Inventory. Level of significance adopted: $5 \%$. ${ }^{*}$ statistically significant difference.

\section{DISCUSSION}

In this study, there was a higher number of female workers with dizziness (92\%) than males (8\%). This finding is consistent with those of other studies, which have also found that dizziness is more prevalent in females. Explanations for the higher prevalence of dizziness in women have largely centered around hormonal changes and increased incidences of migraine. Although this explanation may be valid for the studied sample, the higher number of female participants was also related to the type of work involved in the study, as all participants belonged to the division of nutrition and dietetics, where, in general, more women work than men ${ }^{21}$.

Regarding the VR program conducted with 13 workers of the DND who suffer from dizziness, it was possible to observe its beneficial effect in terms of a reduction of symptoms, mainly verified by the $\mathrm{DHI}$ and the VAS analogue scale.

The DHI has been one of the most used tools when evaluating the effectiveness of VR in different clinical populations. It was one of the indices recommended by the Barany Society Ad Hoc Committee on Vestibular Rehabilitation Therapy in pre- and post-rehabilitation evaluation ${ }^{22}$. A reduction of greater than or equal to 18 points in $\mathrm{DHI}$ scores before and after the intervention is indicative of the intervention being beneficial ${ }^{23}$.
In the present study, this decrease was noted in $42 \%$ of participants, along with a reduction of 13 points in the total final mean. Moreover, it was possible to observe statistically significant differences between the initial and final subscale scores and the total score, indicating an improvement in dizziness symptoms after VR. These results were in agreement with those reported in a study conducted on individuals with peripheral vestibular disease, in the group in which the Cawthorne and Cooksey exercises were used, where $50 \%$ of participants showed reductions in $\mathrm{DHI}$ of greater than 18 points ${ }^{8}$. As in other studies, this index is indicative of improving quality of life 8,16 .

As there are no studies on the application of VR in DND employees, the results of this study reinforce not only what has been demonstrated in other studies on the effectiveness of these programs, but mainly that by using simple exercises that are easy to perform in the working environment, there may be a decrease in a worker's dizziness symptoms. This, in turn, can minimize its harmful effects on quality of life, such as poor concentration, fatigue, irritability, etc. ${ }^{4,5,11,12}$. It may be considered that the statistically significant difference in DHI subscales was the most objective and expressive result of this study and positively indicated the possible implementation of this program among other groups of workers. 
The significant improvement in dizziness after completion of the VR program can also be observed using the VAS, an index that has also frequently been used in other studies with VR ${ }^{11,24}$. In addition to the $\mathrm{DHI}$ and VAS, it was found that in response to the question applied after the VR program ("Do you think that participating in the program has improved your dizziness?"), none of the participants reported that it had "not improved", confirming the positive effect on the participants' perception of dizziness. The perception of improvement by the workers, although more subjective, is not without importance for this study's type of purpose, which ultimately aims to improve quality of life in the workplace. Although an objective, statistical analysis was not possible, one should not disregard the analysis of the VR program's effects in that, from the worker's perspective, $100 \%$ felt it contributed to an improvement in symptoms. Considering the self-perception of the workers involved in this study, the activities proposed in the VR program and applied in the working environment were effective in reducing the degree of dizziness, which further reinforces its application in other areas. Cases where there was no improvement may have been affected by other variables, such as the existence of associated diseases; the possibility that some individuals did not perform the exercises at home, at the prescribed intervals; individual characteristics; or using a generic protocol for all cases. It is suggested that these variables be considered in future studies.

According to the workers' perception, the improvement in dizziness identified by the $\mathrm{DHI}$ and VAS tools did not significantly affect the ability to work. No studies were found in the literature that relate dizziness to the ability to work, although some have reported the effect of dizziness on quality of life ${ }^{12,24}$.

In this study, it is believed that the non-relationship between improvement in dizziness and WAI may be explained by the fact that this index reflects the overall health of the worker, which comprises many different factors ${ }^{25}$. Despite the obvious improvement in dizziness and the direct concomitant effects on physical and mental well-being, the WAI may not have shown significant changes because it relates to workers with work restrictions. In a literature review article ${ }^{14}$, the authors reported that health is undoubtedly the factor that most interferes with the ability to work and that cardiorespiratory fitness and musculoskeletal functioning are the aspects that have the greatest impact on functional capacity because they are directly related to the performance of work demands ${ }^{14,26,27}$.

Finally, the importance of conducting workers' rehabilitation programs in their own working environment should be mentioned, which is a practice oriented toward the worker's health that is now widely advocated. One aspect that should be emphasized in this regard was adherence to the program; due the fact that the program was conducted in the workplace, all of the selected individuals with dizziness attended all VR program sessions, and the majority performed activities at home, contributing to the improvement in dizziness.

Although effects of the VR program on WAI were not identified in this study, it was possible to verify its effectiveness in improving workers' dizziness symptoms by means of the DHI and VAS tools and self-perception, suggesting that this program can be directed to other workplaces.

The identification of, and early intervention in, patients with dizziness in the working environment can assist occupational health services in preventing and minimizing this complaint and contribute to an improvement in workers' health conditions and quality of life.

The study has some limitations, such as the lack of objective evaluation of the vestibular system, the small number of workers and the existence of comorbidities, such as the presence of musculoskeletal diseases. As the focus of the study was to perform all evaluations and the VR program in the working environment and during working hours, using simple, inexpensive and feasible tools, it was possible to verify a decrease in symptoms and the effectiveness of the program. Analysis of this pilot study suggests that application of VR in groups of workers with dizziness and in the working environment can be effective; however, it is believed that the objective evaluation of the vestibular system, for a more accurate diagnosis, and the use of groups with more homogeneous health characteristics may help to improve the program.

Considering that this was a pilot study investigating workers' health and improvements in quality of life, the program produced a reduction in dizziness symptoms, verified by the DHI and the VAS. Despite the evidence of this symptom's improvement, no statistically significant change was observed in relation to the WAl after VR. The study indicates that such programs may be beneficial to the worker and that further studies with other groups of workers in their working environments should be developed. 


\section{CONCLUSION}

In the present pilot study, it was possible to observe the effectiveness of a vestibular rehabilitation program, carried out in the working environment in workers with dizziness complaint, in the Nutrition and Dietetics Division of a public hospital.

\section{REFERENCES}

1. Ogido R, Costa EA, Machado HC. Prevalence of auditory and vestibular symptoms among workers exposed to occupational noise. Rev Saúde Pública. 2009;43(2):377-80.

2. Ganança FF, Castro ASO, Branco FC, Natour J. Interferência da tontura na qualidade de vida de pacientes com síndrome vestibular periférica. Rev Bras Otorrinolaringol. 2004;70(1):94-101.

3. Hueb MM, Feliciano CP. Avaliação diagnóstica das síndromes vertiginosas. Revista do Hospital Universitário Pedro Ernesto. 2012;11(3):24-6.

4. Segarra-Maegaki JA, Taguchi CK. Estudo do benefício da reabilitação vestibular nas síndromes vestibulares periféricas. Pró Fono R Atual. Cientif. 2005;17(1):3-10.

5. Bronstein AM, Golding JF, Gresty MA, Mandalà M, Nuti $D$, Shetye $A$ et al. The social impact of dizziness in London and Siena. J Neurol. 2010;257(2):183-90.

6. Tiensoli LO, Couto ER, Mitre El. Fatores associados à vertigem ou tontura em indivíduos com exame vestibular normal. Rev. CEFAC. 2004;6(1):94-100.

7. McDonnell MN, Hillier SL. Vestibular rehabilitation for unilateral peripheral vestibular dysfunction. Cochrane Database Syst Rev. 2015;1:CD005397.

8. Manso A, Ganança MM, Caovilla HH. Vestibular rehabilitation with visual stimuli in peripheral vestibular disorders. Braz $\mathrm{J}$ Otorhinolaryngol. 2016;82(2):232-41.

9. Taguchi CK, Bohlsen YA. Reabilitação vestibular. In: Boéchat EM, Menezes PL, Couto CM, Frizzo ACF, Scharlach RC, Anastasio ART (orgs). Tratado de audiologia. Rio de Janeiro: Guanabara Koogan; 2015. p. 551-9.

10. Han BI, Song HS, Kim JS. Vestibular rehabilitation therapy: review of indications, mechanisms, and key exercises. J Clin Neurol. 2011;7(4):184-96.

11. Ricci NA, Aratani MC, Caovilla HH, Ganança FF. Challenges in conducting a randomized clinical trial of older people with chronic dizziness: before, during and after vestibular rehabilitation. Contemp Clin Trials. 2015;40:26-34.
12. Tsukamoto HF, Costa VSP, Silva Junior RA, Pelosi GG, Marchiori LLM, Vaz CRS et al. Effectiveness of a vestibular rehabilitation protocol to improve the health-related quality of life and postural balance in patients with vertigo. Int Arch Otorhinolaryngol. 2015;19(3):238-47.

13. Tuomi K, Ilmarinen J, Jahkola A, Katajarinne L, Tulkki A. Índice de capacidade para o trabalho. São Carlos: Editora da Universidade Federal de São Carlos; 2005.

14. Martinez MC, Latorre MRDO, Fischer FM. Capacidade para o trabalho: revisão de literatura. Ciênc. Saúde Coletiva. 2010;15(1):1553-61.

15. Beltrame MT, Magnago TSBS, Kirchhof ALC, Marconato CS, Morais BX. Work ability in hospital housekeeping services and associated factors. Rev Gaucha Enferm. 2014;35(4):49-57.

16. Castro ASO, Cazzola JM, Natour J, Ganança FF. Brazilian version of the Dizziness Handicap Inventory. Pró Fono R Atual. Cientif. 2007;19(1):97-104.

17. Martinez MC, Latorre MRDO. Fatores associados à capacidade para o trabalho de trabalhadores do Setor Elétrico. Cad. Saúde Pública. 2009;25(4):761-72.

18. Cawthorne T. The physiological basis for head exercises. J Chart Soc Physiother. 1944;30:106-7.

19. Cooksey FS. Rehabilitation in vestibular injuries. Proc R Soc Med. 1946;39(5):273-8.

20. Morozetti PG, Ganança CF, Chiari BM. Comparison of different protocols for vestibular rehabilitation in patients with peripheral vestibular disorders. J Soc Bras Fonoaudiol. 2011;23(1)44-50.

21. Bittar RSM, Oiticica J, Bottino MA, Ganança FF, Dimitrov R. Population epidemiological study on the prevalence of dizziness in the city of São Paulo. Braz J Otorhinolaryngol. 2013;79(6):688-98.

22. Cohen HS, Gottshall KR, Graziano M, Malmstrom E, Sharpe MH, Whitney SL. International guidelines for education in vestibular rehabilitation therapy. $J$ Vestib Res. 2011;21(5):243-50.

23. Bayat A, Pourbakht A, Saki N, Zainun Z, Nikakhlagh $\mathrm{S}$, Mirmomeni G. Vestibular rehabilitation outcomes in the elderly with chronic vestibular dysfunction. Iran Red Crescent Med J. 2012;14(11):705-8.

24. Rocha Júnior PR, Kozan ES, Moraes JF, Pereira FG, Moreno AB. Reabilitação vestibular na qualidade de vida e sintomatologia de tontura de idosos. Ciênc. saúde coletiva. 2014;19(8):3365-74. 
25. Martinez MC, Latorre MRDO, Fischer FM. Validity and reliability of the Brazilian version of the Work Ability Index Questionnaire. Rev. Saúde Pública. 2009;43(3):525-32.

26. Sell L, Lund HL, Holtermann A, Søgaard K. The interactions between pain, pain-related fear of movement and productivity. Occup Med. 2014;64(5):376-81.

27. Rostamabadi A, Mazloumi A, Foroushan AR. Work Ability Index (WAI) and its health-related determinants among iranian farmers working in small farm enterprises. J Occup Health. 2014;56(6):478-84. 